\title{
Image Processing Based Toll Automation Technique Using ANPR
}

\author{
${ }^{* 1}$ Dr. P. B. Mane, ${ }^{2}$ Gayatri Bade, ${ }^{3}$ Varsha Patil \\ ${ }^{1}$ Principal, AISSMS Institute of Information Technology, Pune, India \\ Email:pbmane6829@rediffmail.com,gayubade31@gmail.com,varshapatil101@gmail.com
}

Received: $24^{\text {th }}$ November 2018, Accepted: $13^{\text {th }}$ February 2019, Published: $30^{\text {th }}$ June $_{2019}$

\begin{abstract}
For heavy traffic highway roads, particularly in India delay at the toll booths is common scenario. There is a need of an intelligent system that will recognize the vehicle number plate which reduces the amount of time and efforts. Proposed system of toll automation technique using ANPR is designed to ensure a steady flow of traffic. Automatic Number Plate Recognition (ANPR) algorithm based system proposes a methodology in which every vehicle owner will yearly deposit the toll tax in government account if these vehicles are passing through the toll gates equipped with digital camera capturing image of vehicle number plate. Captured images then get processed with Proposed ANPR algorithm. The ANPR algorithm works on steps like extraction of numbers preprocessing via filtering, segmentation and character recognition. Central database is used to store the user's vehicle information. If user is registered account holder, then toll gate will opened and vehicle is passed from toll plaza. The user will be informed about the toll deduction and his account status through the SMS. The performance of algorithm is checked on real images with parameters like success rate, illumination conditions, and tilted position of number plates. Further this system can be utilized for investigation of stolen vehicles.
\end{abstract}

Keywords

ANPR, Extraction, Segmentation, Optical Character Recognition, GSM.

\section{Introduction}

ANPR is an image processing based automatic number plate recognition system used to identify vehicles by only their license numbers on plate. It is automatic toll collection system and free from human interference. The ANPR system consists of the following three steps: Extraction of plate region, character segmentation, and character recognition. ANPR system is used for automatic toll collection at toll booth, automatic ticketing of vehicles at parking area, border control, detection of stolen car, access control in building area, intelligent traffic system and effective control purpose. The main aim of the system is to replace the manual systems with an automated system with proper identification and localization of the number plate information [8].

\section{Literature Survey}

Sarbjit Kaur [8] presents algorithm in which the input vehicle plate image is pre-processed using iterative bilateral filter, adaptive histogram equalization, morphological operations, image subtraction, thresholding, sobel edge detection and boundary box analysis techniques. Mr. G. T. Sutar [2] describes the template matching and correlation technique for Optical character recognition and various image processing techniques such as gray processing, median filtering is implemented and simulated in MATLAB. Farhad Faradji [5] presents a technique which uses sobel mask to extract the vertical edges of input image; histogram analysis and morphological operators are used to find the region of license plate. Serkan Ozbay [4] it presents edge detection and smearing algorithm for plate extraction which finds the exact plate location. Dilation and cross-correlation is used to separate characters as well as find the similarity in recognition procedure. Dinesh Bhardwaj [6] The paper is based on survey of existing ANPR system according to features they used and compare them in terms of processing speed, performance.

Table 1: The Extended Literature Survey is Summarized as:

\begin{tabular}{|l|l|}
\hline \multicolumn{1}{|c|}{ Paper and Author Name } & \multicolumn{1}{|c|}{ Techniques Used. } \\
\hline Anisha Goyal, "Various Techniques for Number & $\begin{array}{l}\text { It is a reviewed of ANPR system which will } \\
\text { Plate Recognition- A Review" June 2016, [7]. } \\
\text { recognize number plates from a distance and also } \\
\text { distorted number plates. It uses optical character } \\
\text { recognition (OCR), split-merge segmentation and } \\
\text { neural network model for recognition purpose. }\end{array}$ \\
\hline $\begin{array}{l}\text { Varsha K Patil, "License Plate Detection System for } \\
\text { Parking in Malls" April 2016, [3]. }\end{array}$ & $\begin{array}{l}\text { System use preprocessing to localized only number } \\
\text { plate region. Algorithm consists of steps like as edge } \\
\text { detection, smoothing, morphological operation like } \\
\text { dilation and erosion. }\end{array}$ \\
\hline
\end{tabular}


Kavya.S, "image processing based toll automation using ANPR” March-May 2015, [1].

It uses a central server which holds the user's information details in the database for matching purpose. The four basic algorithms such as plate localization, pre-processing, segmentation and recognition of the characters.

From literature survey, it is observed that variety of plates such as white characters on black plates, black characters on white background, plate having two-rows, plates having single row, plates having with different character size, plate with different font's size and type, plates with or without special marks exist in practice.

\section{Proposed System Model}

This work proposes a blended software-hardware system which recognizes a license plate automatically and toll collection is done without manual system. Proposed system is based on ANPR work on white background with black character type number plates.

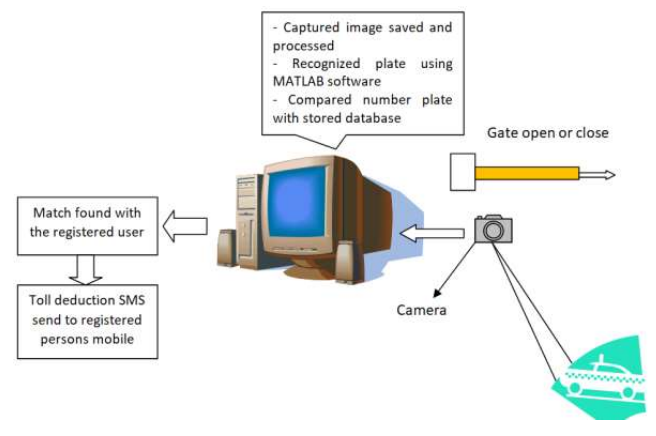

Figure 1: Proposed ANPR System Model

Fig. (1). shows the proposed system model that consists of ANPR algorithm for recognition of vehicle number with the vehicle number plate database stored in central system. If car is detected by IR sensor, vehicle image is captured using digital camera. ANPR algorithm performs the processing operation on it. The recognized number is then compared to stored database. If "Access Granted" for customer's vehicle number plate, toll gate will open and allow vehicle to pass from toll gate. Another case describes that if customer's vehicle has unauthorized/unregistered, toll gate remain closed and "Attention required". Toll amount will be deducted from user's account and user will be informed about toll deduction through the SMS on mobile. System use MATLAB software for image processing purpose.

\section{Image Processing Algorithm used for ANPR System}

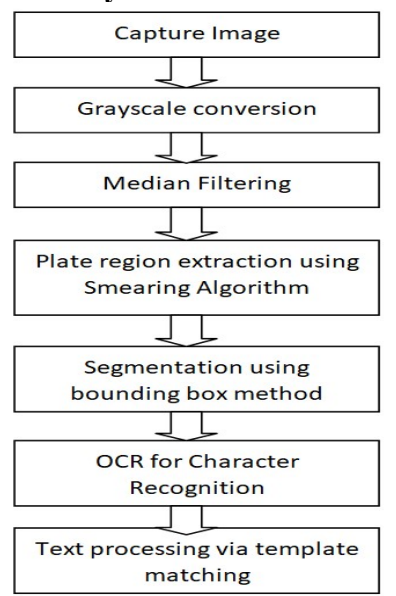

Figure 2: Image Processing Flowchart for ANPR

\section{Image Acquisition:}

System uses a high quality resolution digital camera to acquire images. RGB image is converted to gray scale and all the processing steps are executed on gray scale image. The 8-bit gray value is calculated using formula as shown follows [6]:

A grayscale conversion:

Gray $(i, j)=0.59 * \mathbf{R}(i, j)+0.30 * \mathbf{G}(i, j)+0.11 * \mathbf{B}(i, j)$ 
Where

$\mathrm{R}, \mathrm{G}, \mathrm{B}=$ Red Green Blue components.

Gray $(i, j)=24$-bit color value of each pixel.

Algorithm with following steps is implemented to get image from web camera:

1. Get the object from web camera.

2. Choose the color space and capture the image.

a) Pre-processing:

The following function is used to detect a vehicle number by growing the region and removing noise and horizontal lines.

Algorithm implemented for detection of vehicle number:

1. Take an input image, resize it and convert it into gray.

2. Remove the noise with median filtering.

3. Calculate morphological gradient after opening the image.

4. Get gradient of convolved image in logical 0 or 1 format.

Plate Region Extraction:

Algorithm implemented for cropping of image:

1. Get the image from camera and divide the text into first line and remaining line.

2. Take first line into consideration by cropping the image.

b) Morphological Operations:

Morphological operations are used in pre-processing step which performs various tasks to improve brightness and to remove noises.

Various operations are as follow:

1]. Dilation operation: Dilation adds pixels to the boundaries of objects in an image.

$P \cup Q=\{p \mid p \in P$ or $p \in Q\}$

The union of two sets is denoted as $\mathrm{P} \cup \mathrm{Q}$. (This is the OR operation) Here the resulting set is a group of pixels such that each pixel is an element of $\mathrm{P}$ or $\mathrm{Q}$ or both.

Where

$E=$ member of or belongs to.

$\mathrm{P}=$ binary object, $\mathrm{p}=$ pixels.

2]. Erosion operation: Erosion reduces the number of pixels from the object boundary. The number of pixels removed depends on the size of the structuring element.

$P \cap Q=\{p \mid p \in P$ and $p \in Q\}$

The intersection of two sets is denoted as $\mathrm{P} \cap \mathrm{Q}$. (this is the AND operation). Here the resulting set is a group of pixels such that each pixel belongs to both the sets $\mathrm{P}$ and $\mathrm{Q}$. Erosion combines two sets using vector subtraction of set elements.

Flowchart implemented for the morphological operations is as follows:

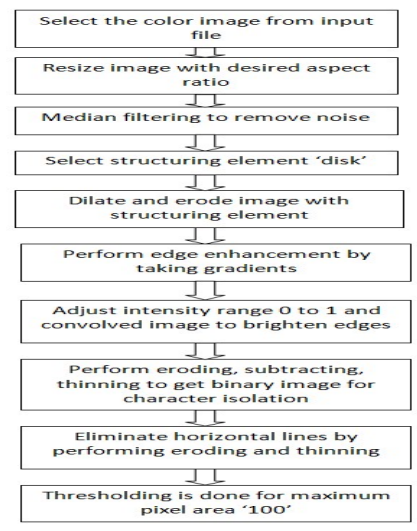

Figure 3: Morphological Operations Flowchart

c) Character Segmentation:

Segmentation is the procedure of partitioning a digital image into multiple segments. Each character is segmented separately. The objects in the image are identified and plotted boxes around them.

Following Algorithm is used for bounding box character segmentation:

- Select the boundary box of order '4' and initialized the number plate string.

- Extract the letters from selected binary image region. 
- These letters are further given to template database for comparison, matching and discarding.

d) Optical Character Recognition:

Optical character recognition is mechanical or electronic conversion of images of handwritten or printed text usually captured by a camera into machine-encoded text. OCR is done with the template matching technique [1]. The matching value of the segmented character is calculated from the templates of the character we store in our database.

e) Central Database:

The central database is an important part of whole system. The user's information is stored in central database and customer is required to register his account with the details about owner, mobile number, vehicle number and account number. Character database stored as a separate image [3]. When registered vehicle passes through toll booth then automatically toll amount will be deducted from user's account and customer receive the information about his account status through SMS to his mobile using GSM modem.

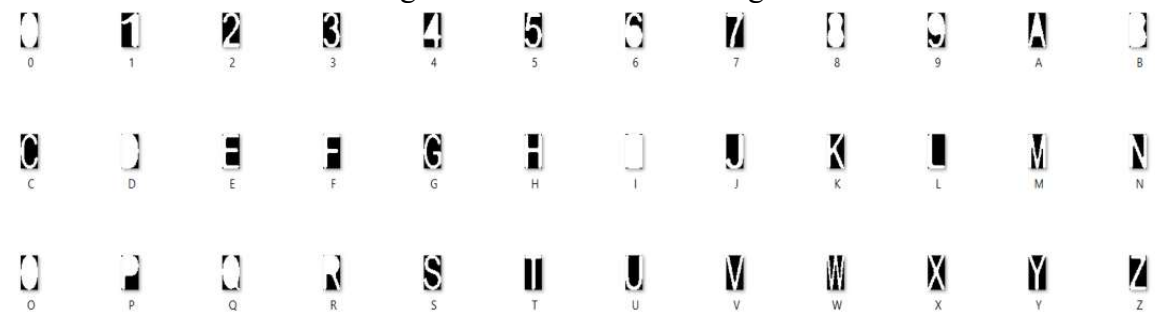

Figure 4: Template Database

Template database consist of letters A-Z and numbers 0-9 as shown in Fig. (4).

Results and Discussion

Fig. (5). Shows the first stage of the output. The digital camera captures the detected image and sends it to ANPR algorithm for processing purpose. The image is converting into grayscale and morphological gradient is calculated after preprocessing via median filtering.

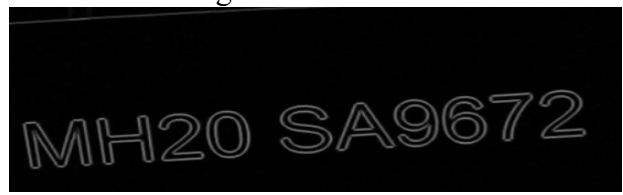

Figure 5: Gray Scale Conversion and Morphological Gradient Operation Done on Image Morphological operations consist of erosion, dilation, subtraction and thinning. These operations are performing in preprocessing step to get binary image for character isolation. Fig. (6). Shows the binary image after noise removing and preprocessing.

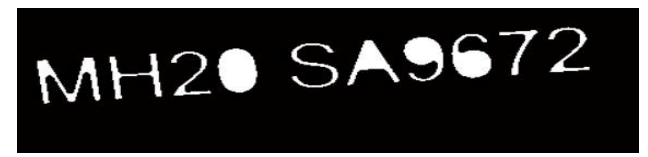

Figure 6: Final Binary Noise Free Image

All noises is removed from image further binary letters are extracted from selected image region. The system uses bounding box analysis of order' 4 ' for character segmentation. Each character and number is separated from each other as shown in Fig. (7).
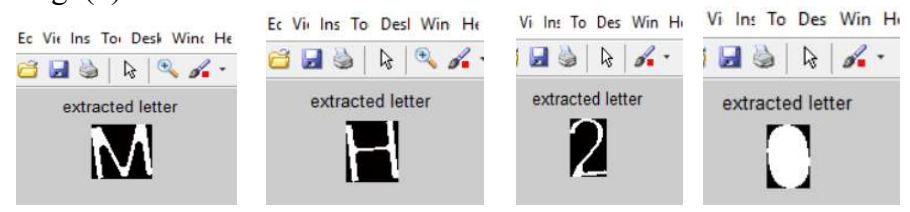

Figure 7: Character Segmented Images

Fig. (8). shows the character recognition output. If customer is registered account holder then information is found in database and amount deduction message is sends to customer's mobile through SMS. 


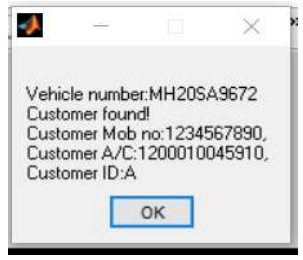

\section{Conclusion}

Figure 8: Final Optical Character Recognition Output

This ANPR system is best method for toll fee collection which can save time and human efforts. It gives accurate result as compare to RFID tag method of toll collection. Our ANPR system works for a specific plate formats such as white background and black font on it which is standard format for number plate. The system used GSM for sending the customer's details on mobile and amount deduction is done if customer is authorized person otherwise toll gate will not be open and next verification will be start. Hence the system is able to save time. Finally advantage is that stolen vehicle or unauthorized person is unable to cross the toll gate.

\section{References}

[1] Kavya.S, Mutturaj Hubballi, Priyanka.B.N, "IMAGE PROCESSING BASED TOLL AUTOMATION USING ANPR" International Journal of Emerging Technologies in Computational and Applied Sciences, 12(2), March-May 2015.

[2] Mr. G. T. Sutar, Prof. Mr. A.V. Shah, "Number Plate Recognition Using an Improved Segmentation" International Journal of Innovative Research in Science, Engineering and Technology, Vol. 3, Issue 5, May 2014.

[3] Varsha K Patil, "License Plate Detection System for Parking in Malls" International Journal of Innovative Research in Computer and Communication Engineering, Vol. 4, Issue 4, April 2016.

[4] Serkan Ozbay, Ergun Ercelebi, "Automatic Vehicle Identification by Plate Recognition" International Journal of Computer and Information Engineering Vol:1, No:9, 2007

[5] Farhad Faradji, Amir Hossein Rezaie, Majid Ziaratban, "A MORPHOLOGICAL-BASED LICENSE PLATE LOCATION" ICIP 2007, IEEE.

[6] Dinesh Bhardwaj, Sunil Mahajan, "Review Paper on Automated Number Plate Recognition Techniques" International Journal of Emerging Research in Management \&Technology, Volume-4, Issue-5, 2015.

[7] Anisha Goyal, "Various Techniques for Number Plate Recognition- A Review" International Journal of Computer Applications, Volume 143 - No.11, June 2016.

[8] Sarbjit Kaur, "An Efficient Approach for Automatic Number Plate Recognition System under Image Processing” International Journal of Advanced Research in Computer Science, Volume 5, No. 6, July-August 2014. 